\title{
A Case Report of a 4-Year-Old Boy with Intradural Spinal Cord Abscess Successfully Treated with Adjuvant Hyperbaric Oxygen Therapy
}

\author{
Ayse SAHIN ${ }^{1}$, Mustafa KILIC ${ }^{2}$, Nazan DALGIC ${ }^{1}$ \\ ${ }^{1}$ University of Health Sciences, Sisli Hamidiye Etfal Research and Education Hospital, Pediatric Infectious Diseases Clinic, Istanbul, Turkey \\ 2University of Health Sciences, Sisli Hamidiye Etfal Research and Education Hospital, Neurosurgery Clinic, Istanbul, Turkey
}

Corresponding author: Ayse SAHIN ayseturgutsahin@gmail.com

\section{ABSTRACT}

Intradural spinal cord abscesses are rare infections in early childhood and usually result from pre-existing congenital anomalies of the spinal column. The formation of abscess may be the result of hematogenous spread. It is treated by surgical and parenteral antibiotic treatment, but some special cases may require additional treatments. This article presents a 4-year-old male patient who was previously operated on for spina bifida (meningocele and tethered cord syndrome) at another hospital. Upon complaints of not being able to walk after one month, he was operated on with the diagnosis of spinal intradural abscess and referred to our clinic to continue his treatment. The patient underwent an emergency operation when his new spinal magnetic resonance imaging (MRI) at our hospital showed progression of intradural abscess. Due to no regression of neurological deficits in the follow-up and the risk of another operation, antimicrobial treatment as well as hyperbaric oxygen therapy (HBOT) was planned. At the end of 20 HBOT treatment sessions, the patient started to walk with support and the antibiotic treatment was completed in six weeks. In cases where surgical and antimicrobial treatments have failed, HBOT should be considered as an additional treatment method in children with spinal abscess.

KEYWORDS: Abscess, Child, Hyperbaric oxygen therapy, Spinal cord

\section{INTRODUCTION}

I ntradural abscess of the spinal cord is known to be a rare clinical entity. In adults, pathophysiology of this disease is chiefly hematogenous spread from a cardiopulmonary source. In children, hematogenous spread from a variety of sources occurs, but pre-existing congenital defects in spinal cord development are the more common means by which infection develops (12).

Hyperbaric oxygen therapy (HBOT) is a treatment in which the patient breathes $100 \%$ oxygen intermittently while inside a treatment chamber at a pressure that is higher than sea level pressure (10). HBOT is known as the primary medical treatment for decompression sickness and arterial gas embolism. HBOT is also a treatment method for many health problems such as necrotizing soft tissue infections, clostridial myonecrosis (gas gangrene), necrotizing fasciitis, acute traumatic ischemia, chronic diabetic and non-diabetic hypoxic problem wounds, chronic refractory osteomyelitis, radiation tissue damage, compromised skin grafts, and some types of intracranial abscesses $(2,5,8)$.

In this study, we present the successful treatment of a 4-yearold male patient who had spinal lumbosacral intradural abscess and underwent surgery and antimicrobial treatment alongside HBOT which was the first case in literature.

\section{CASE REPORT}

A four-year old male patient who had been diagnosed with spina bifida (meningocele and tethered cord syndrome) and operated by a neurosurgeon started to complain of pain in his legs and waist, after one month the operation. 
On his examination, he could not walk, his legs could not move and his sensation was lost. A vasculitic rash on the midfoot was detected by the pediatric rheumatologist and colchicine treatment was initiated after obtaining blood for vasculitis panels (anti-ds DNA, MPO, p-ANCA) and Familial Mediterranean Fever (FMF) mutation evaluation.

After a steady increase in the patient's leg pain, restricted lower limb movements and increased sensory loss, a spinal magnetic resonance imaging (MRI) scan revealed a spaceoccupying lesion and was suspected to be an abscess. Drainage of the abscess at the central location was attempted by lumbar puncture but was unsuccessful. An intradural abscess was then diagnosed and intravenous administration of vancomycin and meropenem were started under operating room conditions.

The patient was referred to our hospital due to symptoms during follow-up and he was conscious with moderate general condition on physical examination. Sensory loss and motor loss were present in the upper extremities while bilateral lower extremity proximal muscle strength was graded $0 / 5$ and distal muscle strength was graded 0/5. Patellar and Achilles reflexes were bilaterally absent and other system examinations were normal. Blood parameters showed only an elevated C-reactive protein level of $92.9 \mathrm{mg} / \mathrm{dL}$ (normal $<5 \mathrm{mg} / \mathrm{dL}$ ) while other laboratory findings were normal. Colchicine treatment was discontinued due to normal vasculitis and FMF tests but meropenem was continued.

The spinal MRI scan of the patient at our hospital showed elongation in the paravertebral soft tissues extending from the T10 level to the sacral region (Figure 1A, B), and there were findings compatible with an elongated abscess under the skin. In the abscess culture obtained from the patients' emergency abscess drainage, Extended Spectrum BetaLactamase (EBSL) positive Escherichia coli (E. coli) was grown and it was sensitive to meropenem. On the $18^{\text {th }}$ day of antibiotic treatment, a repeated MRI scan showed a progressing abscess (Figure 2A, B), and physical examination revealed sensory and motor deficits in the patient's lower extremity. A second operation was considered to be risky and simultaneous corticosteroids ( $2 \mathrm{mg} / \mathrm{kg} /$ day) and HBOT were planned to be administered with the aim of reducing intradural inflammation while antibiotic therapy was ongoing. HBOT was started at 2.5 ATA (absolute atmospheres), 120 minutes/ day. On the $5^{\text {th }}$ day of HBOT treatment, the patient began to withdraw his leg in response to a painful stimulus. The patient could not lift his legs on the $8^{\text {th }}$ day of treatment, but could gather strength to turn from the right and left. On the $15^{\text {th }}$ day of treatment, he could sit on his own and he could stand and walk with support on the $21^{\text {st }}$ day. HBOT was administered for a total of 20 sessions and there were no complications during the treatment. No intradural contrast-enhancing lesion was found on the follow-up MRI at 37 days after admission (Figure $3 \mathrm{~A}, \mathrm{~B})$. The physical examination performed during the followup of the first month was completely normal and the patient could walk without support.

\section{- DISCUSSION}

Spinal intradural abscess is an extremely rare condition, especially in pediatric cases (4). latrogenic spinal infections (ISE) are considered possible complications that could arise after spinal surgical procedures $(3,9)$. ISEs are seen at rates of $0.7 \%$ to $16 \%$, depending on the procedure and the comorbidities present in the preoperative period (9).

Neurological deficits have been reported in $89 \%$ of pediatric patients with spinal abscess. Paralysis, paresthesia, urinary retention, and anal incontinence are the most commonly observed (12). The most frequent determinant in spinal abscess is Staphylococcus aureus, and E. coli can also be detected $(11,12)$. In our case, the patient could not walk and the loss of sensation in the lower extremity progressed one month after the operation performed due to spina bifida (meningocele and tethered cord syndrome). In the abscess culture of our patient, EBSL (+) E. coli was detected and was sensitive to the empirically initiated meropenem, which was continued and completed to 6 weeks.
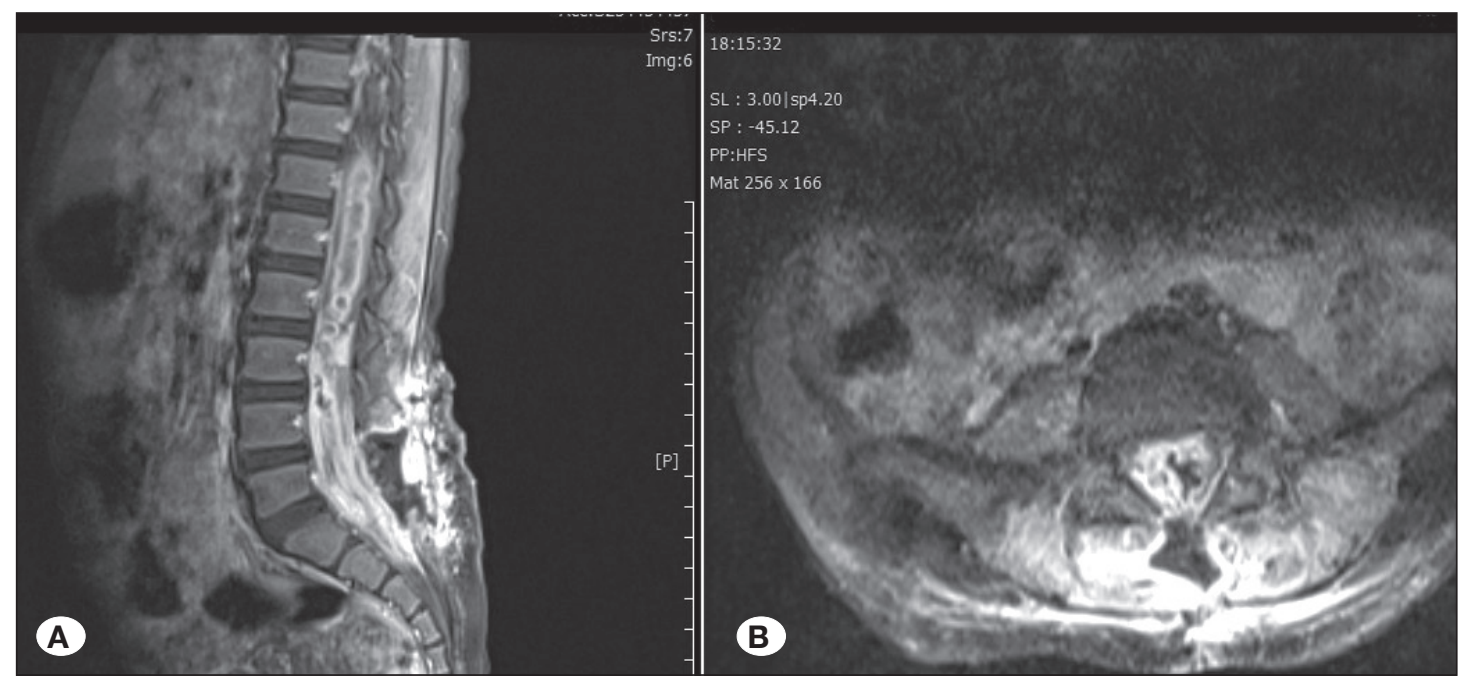

Figure 1: Sagittal (A) axial (B) T1-weighted contrast-enhanced lumbosacral MRI of a 4-year-old male patient shows heterogeneous contrast enhancement starting from T10 and progressing to $\mathrm{S} 2$ level compatible with intradural abscess. 
HBOT is considered as a treatment for many health problems in adults and children. It is reported that HBOT can be used as a safe treatment method for iatrogenic cranial and spinal infections after brain surgery $(6,7,9)$. Oxygen has a direct antimicrobial effect, particularly on anaerobes, and the oxygen in hypoxic tissues can lead to restoration of white blood cells and antimicrobial functions (1). A 68-year-old male patient diagnosed with hypopharyngeal carcinoma who underwent chemoradiotherapy was later treated with HBOT due to the high risk of surgical treatment for the cervical spine osteomyelitis and epidural abscess and a large defect in the posterior hypopharyngeal region that developed after the initial treatment. HBOT has been reported to be a useful treatment method for cervical spinal osteomyelitis and epidural abscesses which are risky for surgery (15). In a study by Topuz et al. (14), HBOT in addition to chemotherapy was administered to 16 out of 51 patients diagnosed with spinal tuberculosis and earlier clinical and radiological improvement was detected.
In a study on adult patients, HBOT in addition to conservative treatment alongside percutaneous drainage was administered to 23 patients aged between 45 and 85 years with an iliopsoas abscess due to pyogenic spondylitis. Clinical and radiological improvement was achieved immediately after the treatment, and no recurrence was observed in the follow-ups. In these cases, the use of HBOT was reported to be an effective treatment (13).

In a study of five children aged between 11 and 17 years diagnosed with brain abscess, HBOT was administered in addition to surgery and antibiotic treatment. Clinical followup showed no symptoms at the end of 6 months and no recurrence at the end of 21 months. The administration of HBOT together with antibiotherapy and drainage, the duration of intravenous antibiotics and its safety in critical patients have been reported in this study (12). Adjuvant HBOT therapy was successfully applied for retrocerebellar arachnoid cyst and hydrocephalus to an 8-month case of epidural abscess in the posterior fossa after repeated surgical operations. There was
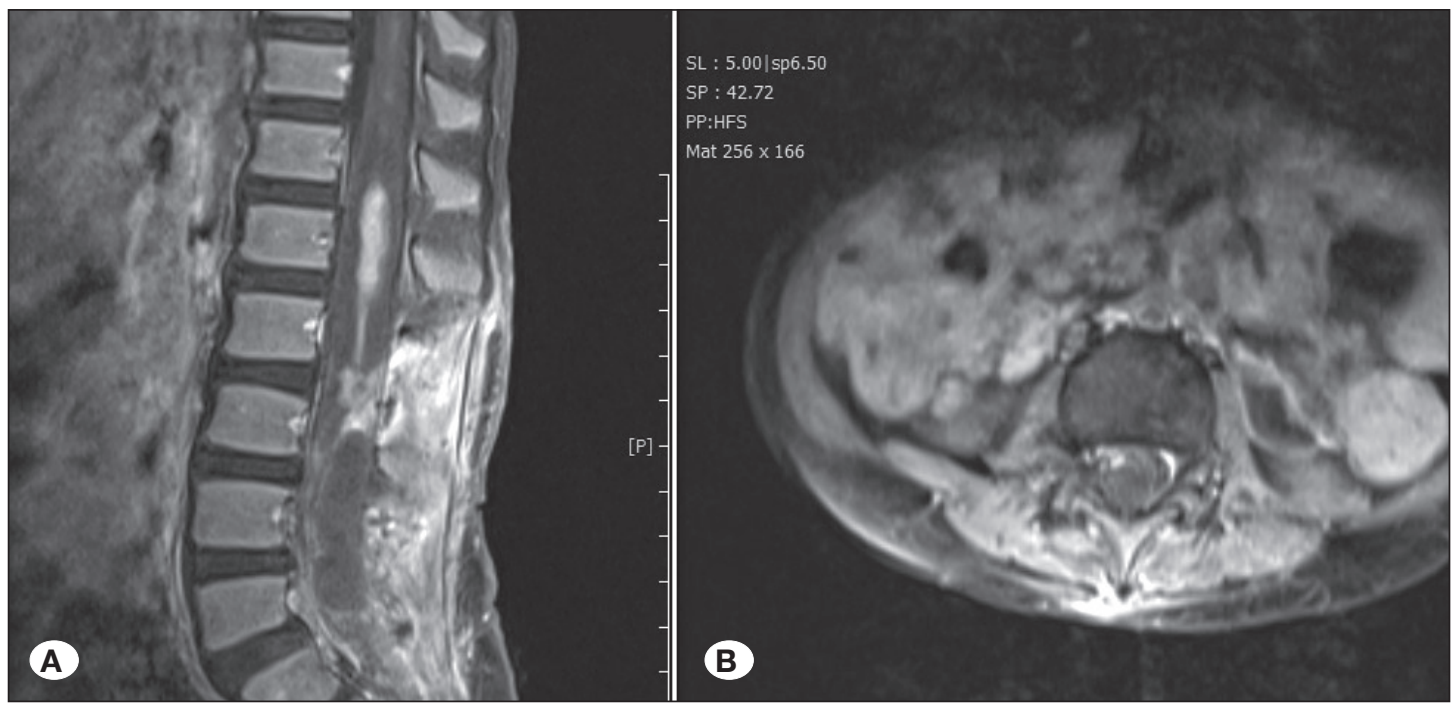

Figure 2: Sagittal (A) and axial (B) T1weighted contrastenhanced lumbar MRI after surgery and before HBOT shows residual heterogeneously enhancing intradural abscess extending from T12 to L3-4.
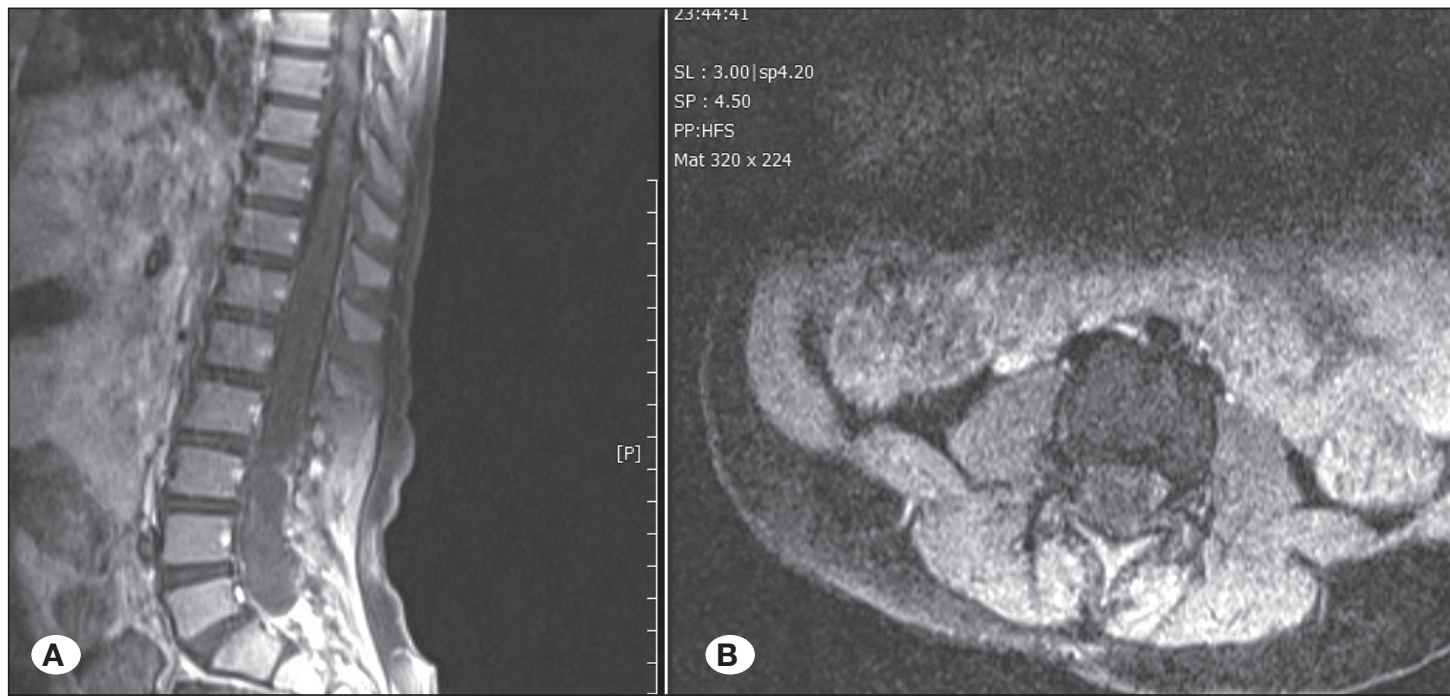

Figure 3: Sagittal (A) and axial (B) T1weighted contrast enhanced lumbar MRI reveals no intradural contrast-enhancing lesion after HBOT. 
no need for a ventriculoperitoneal shunt and bone removal process (1). Minimal side effects were recorded for the HBOT treatment and it involved momentary pressure equalization problems. After our patient had been operated on twice for abscess drainage while using antibiotic treatment, a third operation was considered to be risky. HBOT was administered and successfully completed. No side effects were observed in our patient.

\section{- CONCLUSION}

In case of unresponsiveness to antibiotics and surgical treatment, which is the main treatment approach for critical pediatric patients with spinal intradural abscess and neurological deficit, the viable HBOT method may be administered as an additional treatment strategy.

\section{REFERENCES}

1. Baechli H, Schmutz J, Mayr JM: Hyperbaric oxygen therapy (HBO) for the treatment of an epidural abscess in the posterior fossa in an 8-month-old infant. Pediatr Neurosurg 44(3): 239242, 2008

2. Frawley GP, Fock A: Pediatric hyperbaric oxygen therapy in Victoria, 1998-2010. Pediatr Crit Care Med 13(4): e240-e244, 2012

3. Hasan MY, Kumar KK, Lwin S, Lau LL, Kumar N: Cervical intradural abscess masquerading as an epidural collection. Global Spine J 3(4): 249-252, 2013

4. Karatay M, Koktekir E, Celik H, Erdem Y, Sertbas Bayar MA: Spinal intradural abscess caused by hematogenous spread of Prevotella oralis in a 3-year-old child with an asymptomatic congenital spinal abnormality. Spinal Cord 53 Suppl 1:13-15, 2015

5. Kurschel S, Mohia A, Weigl V, Eder HG: Hyperbaric oxygen therapy for the treatment of brain abscess in children. Childs Nerv Syst 22(1): 38-42, 2006
6. Larsson A, Engström M, Uusijarvi J, Kihlström L, Lind F, Mathiesen T: Hyperbaric oxygen treatment of postoperative neurosurgical infections. Neurosurgery 50(2): 287-296, 2002

7. Larsson A, Uusijarvi J, Lind F, Gustavsson B, Saraste H: Hyperbaric oxygen in the treatment of postoperative infections in paediatric patients with neuromuscular spine deformity. Eur Spine J 20(12): 2217-2222, 2011

8. Nakahara K, Yamashita S, Katsumasa I, Shindo S, Suga T, Ueda A, Honda S, Hirahara T, Watanabe M, Yamashita T, Maeda Y, Yonemochi Y, Takita T, Ando Y: Drastic therapy for Listerial brain abscess involving combined hyperbaric oxygen therapy and antimicrobial agents. J Clin Neurol 10(4): 358362, 2014

9. Onen MR, Yuvruk E, Karagöz G, Naderi S: Efficiency of hyperbaric oxygen therapy in iatrogenic spinal infections. Spine 40 (22): 1743-1748, 2015

10. Shah J: Hyperbaric oxygen therapy. J Am Col Certif Wound Spec 2(1): 9-13, 2010

11. Shtaya A, Hettige S: Disco vertebral osteomyelitis causing intradural spinal abscess with cauda equina compression. $\mathrm{Br}$ J Neurosurgry 31(3): 1-3, 2017

12. Simon JK, Lazareff JA, Diament MJ, Kennedy WA: Intramedullary abscess of the spinal cord in children: A case report and review of the literature. Pediatr Infect Dis J 22(2): 186-192, 2003

13. Tofuku K, Koga H, Komiya S: Percutaneous drainage combined with hyperbaric oxygen therapy for pyogenic spondylitis with iliopsoas abscess. Asian Spine J 8(3): 253-259, 2014

14. Topuz K, Kutlay AM, Simşek H, Colak A, Kaya S, Demircan $\mathrm{MN}$ : Effect of hyperbaric oxygen therapy on the duration of treatment of spinal tuberculosis. J Clin Neurosci 16(12): 15721577, 2009

15. Ueki Y, Watanabe J, Hashimoto S, Takahashi S: Cervical spine osteomyelitis and epidural abscess after chemoradiotherapy for hypopharyngeal carcinoma: A case report. Case Rep Otolaryngol 2014:141307, 2014 